OPEN ACCESS

Edited by: Changqiang Wu, North Sichuan Medical College, China

Reviewed by:

Haoran Sun,

Tianjin Medical University General

Hospital, China

Gang Huang,

Gansu Provincial Hospital, China

*Correspondence:

Bin Song

songlab_radiology@163.com

Hao Zeng

kucaizeng@163.com

${ }^{\dagger}$ These authors have contributed equally to this work

Specialty section:

This article was submitted to

Cancer Imaging and

Image-directed Interventions,

a section of the journal

Frontiers in Oncology

Received: 17 December 2020

Accepted: 14 January 2021

Published: 26 February 2021

Citation:

Cheng X, Xu J, Chen Y, Liu Z, Sun G,

Yang $L$, Yao J, Zeng $H$ and Song $B$ (2021) Is Additional Systematic Biopsy Necessary in All Initial Prostate Biopsy Patients With Abnormal MRI?

Front. Oncol. 11:643051.

doi: 10.3389/fonc.2021.643051

\section{Is Additional Systematic Biopsy Necessary in All Initial Prostate Biopsy Patients With Abnormal MRI?}

\author{
Xueqing Cheng ${ }^{1 \dagger}$, Jinshun $\mathrm{Xu}^{2 \dagger}$, Yuntian Chen ${ }^{1}$, Zhenhua Liu ${ }^{3}$, Guangxi Sun ${ }^{3}$, \\ Ling Yang ${ }^{1}$, Jin Yao ${ }^{1}$, Hao Zeng ${ }^{3 *}$ and Bin Song ${ }^{1 *}$

\begin{abstract}
${ }^{1}$ Department of Radiology, West China Hospital, Sichuan University, Chengdu, China, ${ }^{2}$ Department of Ultrasound, West China Hospital, Sichuan University, Chengdu, China, ${ }^{3}$ Department of Urology, West China Hospital, Sichuan University,
\end{abstract} \\ Chengdu, China
}

Purpose: To determine whether additional systematic biopsy is necessary in all biopsy naïve patients with MRI visible lesions by taking PI-RADS score and prostate volume into consideration.

Materials and Methods: Patients who underwent combined systematic biopsy (SB) and cognitive MRI-targeted biopsy (TB) in our hospital between May 2018 and June 2020 were retrospectively reviewed. The detection rate of clinical significant prostate cancer (csPCa), biopsy grade group (GG) concordance, and disease upgrading rate on radical prostatectomy were compared between SB and TB and further stratified by PI-RADS v2.0 category and prostate volume.

Results: A total of 234 patients were analyzed in this study. TB alone detected more csPCa and less clinically insignificant prostate cancer (cisPCa) than SB alone in the whole cohort (57.3 vs 53\%, $\mathrm{P}=0.041 ; 3.8$ vs $7.7 \%, \mathrm{P}=0.049$ respectively). The additional SB indicated only a marginal increase of csPCa detection but a remarkable increase of cisPCa detection compared with targeted biopsy (59.4 vs 57.3\%, $\mathrm{P}=0.064$; 3.8 vs $7.7 \%, \mathrm{P}=$ 0.012). As stratified by PI-RADS category, the difference of csPCa detection rate between TB and SB was not significant either in PI-RADS 5 subgroup (83.8 vs $76.3 \%, \mathrm{P}=0.07$ ) or in PI-RADS 3-4 subgroup (43.5 vs 40.9\%, $P=1.0$ ). Additional SB decreased the rate of disease upgrading on radical prostatectomy (RP) than TB alone in PI-RADS 3-4 subgroup (14.5 vs 25.5\%, $P=0.031$ ) other than PI-RADS 5 subgroup (6 vs $6 \%, P=1.0$ ). When stratified by prostate volume (PV), TB alone detected more csPCa than SB in small prostate ( $P V<30 \mathrm{ml}$ ) group (81.0 vs $71.0 \%, P=0.021$ ) but not in large prostate ( $P V \geq$ $30 \mathrm{ml}$ ) group (44.0 vs $42.7 \%, \mathrm{P}=0.754$ ). The additional SB did not significantly decrease the rate of disease upgrading on RP than TB alone in either small or large prostate (6.4 vs $8.5 \%, P=1.0 ; 13.8$ vs $22.4 \%, P=0.063$ ).

Conclusion: The combination biopsy method was no superior than targeted biopsy alone in PI-RADS 5 or in small volume prostate subgroup.

Keywords: prostate cancer, prostate biopsy, targeted biopsy, MRI, PI-RADS 


\section{INTRODUCTION}

The standard 10 or 12 cores systematic transrectal ultrasound biopsy (TRUS) was the most common diagnostic method for men suspected with prostate cancer (PCa) on the basis of elevated prostate-specific antigen (PSA) level or an abnormal digital rectal examination (1). But this random sampling strategy is lacking reliability and associated with missed clinically significant $\mathrm{PCa}$ (csPCa) and substantial inaccurate risk stratification $(2,3)$. In addition, systematic biopsy can inadvertently detect indolent PCa causing overdiagnosis and eventually overtreatment.

With recent advances, prostate multiparametric magnetic resonance imaging (mpMRI) has been widely used as a triage test before biopsy in clinical practice, which could reduce unnecessary prostate biopsies (4). Meanwhile, MRI-targeted biopsy allows better sampling of cancer through accurate localization of suspicious prostate lesions just as other solid organs tumors (5-10). Many studies demonstrated that MRItargeted biopsy improved csPCa detection and cancer risk stratification compared with systematic biopsy $(6,9,11-14)$. Although the NICE and the EAU guidelines recommend combined targeted and systematic biopsies in case of positive MRI findings, particularly for repeat biopsies $(15,16)$, questions about the necessity for additional systematic biopsy still persist. As reported, for every one additional csPCa detected, 60 patients need systematic biopsy in addition to targeted biopsy (17). While this combined biopsy strategy leads to a large number of cores being taken, thus further increasing the risk of complication and injury inherent to prostate biopsies as well as the economic burden. Therefore, it is of clinical importance to assess the clinical implications of targeted biopsy with additional systematic biopsy.

Prostate volume and PI-RADS category are the major two factors taken into consideration to perform initial prostate biopsy in men with suspected prostate cancer based on elevated PSA, but whether they could be incorporated to the selection of optimal biopsy method is unknown. In this study, we assessed the use of cognitive MRI guided targeted, systematic, or combined prostate biopsy in an attempt to determine whether the systematic biopsy is necessary in all initial biopsy naïve patients with abnormal MRI and whether PI-RADS score and prostate volume should affect the type of biopsy method that is selected.

\section{MATERIALS AND METHODS}

\section{Patients}

This study was a retrospective study approved by the institutional review board with a waiver of informed consent. Patients suspected with prostate cancer for elevated prostatespecific antigen (PSA) level or abnormal digital rectal examination and subsequently underwent combined systematic biopsy and cognitive MRI-targeted biopsy in our hospital between May 2018 and June 2020 were included. The prebiopsy mpMRI indicated suspicious prostate lesions (PIRADS $\geq 3$ ). The exclusion criteria include: a) previous prostate biopsy, prostate surgery, or neoadjuvant hormonal therapy before biopsy; b) missing PSA or PSA $>100 \mathrm{ng} / \mathrm{ml}$; c) MRI not performed at our institution; $d$ ) interval between MRI and biopsy longer than 6 months.

\section{Image Acquisition and Interpretation}

Multiparametric MRI was performed using 3.0-T MRIs (Magnetom Skyra, Siemens) with phased-array body surface coil. All images were obtained with $3-\mathrm{mm}$ section thickness. T2-weighted images in the sagittal, coronal, and axial planes, diffusion weighted images (b value up to $1,500 \mathrm{~s} / \mathrm{mm}^{2}$ ) in the axial plane, and dynamic contrast-enhanced images were acquired according to the international prostate MRI guidelines (18). MRI lesions were assigned a Prostate Imaging Reporting and Data System Version 2 (PI-RADS v2.0) score of 1 to 5. Two radiologists with respectively 3 and 10 years of experience in abdominal imaging read the images for each patient separately (18). Radiologists were not blinded to clinical information. The lesion with the highest PI-RADS score on mpMRI was defined as the index lesion. If there were two or more foci of equally high PI-RADS score, then the largest one was designated the index lesion.

\section{Biopsy Technique and Histological Evaluation}

All prostate biopsies were performed transperineally under local anesthesia using TRUS guidance with a bi-planar ultrasound probe (BK Medical, USA). A standard 10 or 12 cores systematic biopsy (SB) was obtained including transitional, peripheral, anterior zone from base to apex followed by cognitive MRIguided targeted biopsy (TB). The MR images were available for direct review during the biopsy. Each lesion with a PI-RADS score of 3-5 was biopsied using two or three cores (a maximum of five cores per patient). All biopsy procedures were performed by two experienced urologists with more than 10 years of experience in prostate biopsy, and guided by an experienced urological radiologist (with more than 10 years' experience in TRUS guiding prostate biopsy). Where a lesion was visible at TRUS, it was targeted by using the core for the relevant prostate zone (no additional cores were performed).

All prostate biopsy cores were individually labeled and were analyzed by two dedicated uropathologists. For patients diagnosed as PCa, the number of positive cores, proportion of cancer involvement, as well as grade group (GG) and Gleason score (GS) were determined using the 2014 International Society of Urologic Pathology (ISUP) criteria (19). A GG $\geq 2$ (GS $\geq 3+4$ ) was defined as clinically significant prostate cancer (csPCa), whereas others were defined as clinically insignificant prostate cancer (cisPCa) (19).

\section{Statistical Analysis}

Comparisons of categorical variable were performed using the chi-square test and continuous variables were evaluated with the Student $t$ test after evaluating normality of the data using a onesample Kolmogorov-Smirnov test. One way ANOVA was used 
for comparison of continuous variables between groups unless the data were not normally distributed, in which case the Kruskal-Wallis test was used. Wilcoxon's matched-pairs signed-rank test was used to compare number of biopsy cores, number of positive cores, and percentage of cancer involvement. The McNemar test was used to evaluate differences in cancer detection rates and upgrading rates on radical prostatectomy between each biopsy method. A $P<0.05$ was considered to indicate statistical significance. The statistical analysis was performed using SPSS (ver. 19.0; SPSS Inc., Chicago, IL, USA).

\section{RESULTS}

\section{Clinical Characteristics}

There were 290 patients performed systematic combined with targeted biopsy $(\mathrm{TB}+\mathrm{SB})$, and 56 of them were excluded because of prior prostate biopsy or surgery $(n=33)$, MRI not performed at our institution $(\mathrm{n}=5)$; missing PSA or PSA $>100 \mathrm{ng} / \mathrm{ml}(\mathrm{n}=$ 10), and duration between biopsy and MRI longer than 6 months $(\mathrm{n}=8)$. Finally, there were 234 patients included in this study (Figure 1), $48(20.5 \%)$ of them had a PI-RADS v2.0 score of 3 , $106(45.3 \%)$ had a score of 4 , and $80(34.2 \%)$ had a score of 5 . The median number of MRI lesions detected was 1 , with a median of 3 targeted cores and 12 systematic cores taken per patient. Patient demographics are shown in Table $\mathbf{1 .}$

\section{Targeted Versus Systematic Cancer Detection and Risk Stratification for the Whole Cohort}

The two biopsy methods were compared in terms of the highest GG detected per patient, and the highest GG that was detected by
TABLE 1 | Patient characteristics.

\begin{tabular}{|c|c|}
\hline Characteristics & Total \\
\hline No. of patients & 234 \\
\hline Age (y) & $66.3 \pm 8.95$ \\
\hline PSA (ng/ml) & $12.5 \pm 9.94$ \\
\hline Prostate volume on MRI (ml) & $42.2 \pm 23.65$ \\
\hline No. of MRI lesions & $1(1,2)$ \\
\hline Maximum diameter of index lesion (cm) & $1.5 \pm 0.61$ \\
\hline \multicolumn{2}{|l|}{ PI-RADS Score } \\
\hline 3 & $48(20.5 \%)$ \\
\hline 4 & $106(45.3 \%)$ \\
\hline 5 & $80(34.2 \%)$ \\
\hline \multicolumn{2}{|l|}{ Combined biopsy results } \\
\hline No cancer & 77 \\
\hline GG1 (GS $3+3)$ & 18 \\
\hline GG2 (GS $3+4)$ & 61 \\
\hline GG3 (GS 4 + 3) & 50 \\
\hline GG4 (GS $4+4 / 3+5)$ & 13 \\
\hline GG5 (GS 4 + 5) & 15 \\
\hline No. of cores on TB & $3(3,5)$ \\
\hline No. of cores on SB & $12(12,12)$ \\
\hline No. of positive samples on TB & $2(0,3)$ \\
\hline No. of positive samples on SB & $1(0,4)$ \\
\hline Cancer involvement on TB (\%) & $30(15,60)$ \\
\hline Cancer involvement on SB (\%) & $17.5(10,30)$ \\
\hline
\end{tabular}

either biopsy method was considered as the GG detected on combined biopsy as shown in Table 2. There was a significant difference in the number of positive cores and percentage of cancer involvement of positive core (Table 1, both $\mathrm{P}<0.001$ ). Among 234 patients who underwent combined biopsy, 139 (59.4\%) were diagnosed of csPCa, 18 (7.7\%) were cisPCa, and $77(33.5 \%)$ were not cancer. When each method was used alone, the detection rate of csPCa decreased to $57.3 \%$ (134 of 234 men) for TB and 53\% (124 of 234 men) for SB (Figure 2). TB had a
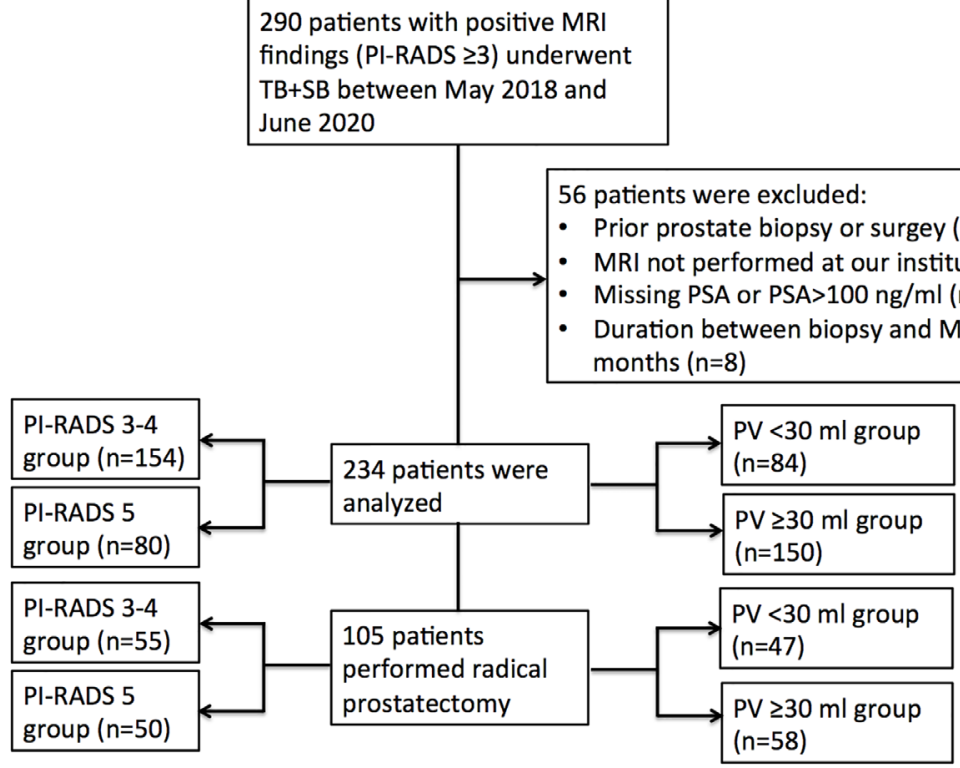

56 patients were excluded:

- Prior prostate biopsy or surgey $(n=33)$

- MRI not performed at our institution $(n=5)$

- Missing PSA or PSA $>100 \mathrm{ng} / \mathrm{ml}(\mathrm{n}=10)$

- Duration between biopsy and MRI > 6 months $(n=8)$

FIGURE 1 | Flow chart 
TABLE 2 | Cross-tabulation of highest grade group detected by biopsy method.

No. of Patients in Grade Group (GG) with SB

\begin{tabular}{lcccccccc} 
& & No cancer & GG1 & GG2 & GG3 & GG4 & GG5 & Total \\
\hline No. of & No cancer & 77 & 10 & 4 & 0 & 0 & 0 & 91 \\
Patients in & GG1 & 3 & 5 & 1 & 0 & 0 & 0 & 9 \\
Grade & GG2 & 6 & 3 & 47 & 0 & 1 & 0 & 57 \\
Group (GG) & GG3 & 3 & 0 & 9 & 38 & 0 & 1 & 51 \\
with TB & GG4 & 2 & 0 & 0 & 3 & 7 & 2 & 14 \\
& GG5 & 1 & 0 & 0 & 3 & 0 & 8 & 12 \\
& Total & 92 & 18 & 61 & 44 & 8 & 11 & 234
\end{tabular}

The blue shading means upgrading by TB, the grey shading means upgrading by both biopsy methods, and the green shading means upgrading by SB.

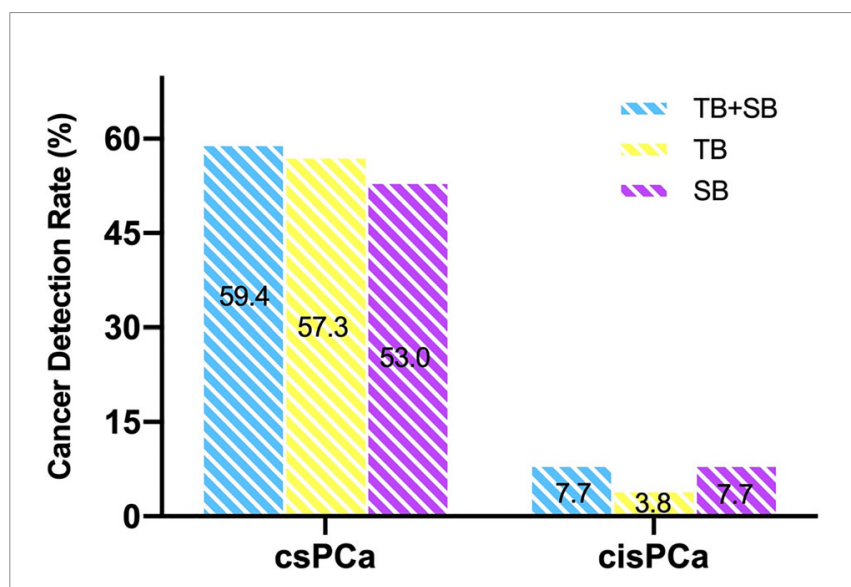

FIGURE 2 | Comparison of csPCa and cisPCa detection rate between TB+SB, TB alone, and SB alone in the whole cohort.

significantly greater csPCa detection rate than $\mathrm{SB}(\mathrm{P}=0.041)$, and similar csPCa detection rate with $\mathrm{TB}+\mathrm{SB}(\mathrm{P}=0.063)$. While, the cisPCa detection rate of $\mathrm{TB}$ was significantly lower than $\mathrm{TB}+\mathrm{SB}$ and $\mathrm{SB}(\mathrm{P}=0.012, \mathrm{P}=0.049$ respectively; Figure 2). Among the 14 (6.0\%) prostate cancer missed by TB, only 4 (28.6\%) was GG2, and the other was all GG1 with no more than 2 cores positive on SB (Table 2). But $\mathrm{SB}$ alone missed $6 \mathrm{GG} \geq 3,6 \mathrm{GG} 2$, and 3 GG1 cancers.

Among 157 patients diagnosed as prostate cancer by combined biopsy, 105 (66.9\%) had concordant grade group between TB and SB, 33 (21\%) patients had upgraded GG on TB over SB, and 19 (12.1\%) had upgraded GG on SB over TB. Omission of SB would lead a reclassification to lower risk stratification in three patients. But the omission of targeted biopsy would make 9 prostate cancers reclassified to lower stratification (Table 2).

\section{Relationship of PI-RADS, Prostate Volume With CDR}

As shown in Figure 3, the csPCa detection rate increased significantly with a greater PI-RADS score $(\mathrm{P}<0.001)$, with no significant difference between biopsy methods. This finding was not seen in cisPCa detection by any of $\mathrm{TB}+\mathrm{SB}(\mathrm{P}=0.182)$, TB $(\mathrm{P}=0.565)$, and $\mathrm{SB}(\mathrm{P}=0.259)$. Taking prostate volume into consideration, there was a significant trend in increased detection of csPCa with decreased prostate volume by all methods (Figure 4, all $\mathrm{P}<0.001$ ).

\section{Targeted Versus Systematic Cancer Detection and Risk Stratification Stratified by PI-RADS Score}

To evaluate cancer detection and risk stratification by PI-RADS score, patients were split into PI-RADS $5(\mathrm{n}=80)$ and PI-RADS 3-4 group $(\mathrm{n}=154)$. The conjunction of SB detected additional one and four csPCa in PI-RADS 5 and PI-RADS 3-4 group respectively, but didn't lead to a higher csPCa detection rate compared than $\mathrm{TB}$ alone in both groups $(\mathrm{P}=1.0, \mathrm{P}=0.125$ respectively, Table 3 ). Meanwhile, the difference of csPCa detection rate between $\mathrm{TB}$ and $\mathrm{SB}$ was not significant either in

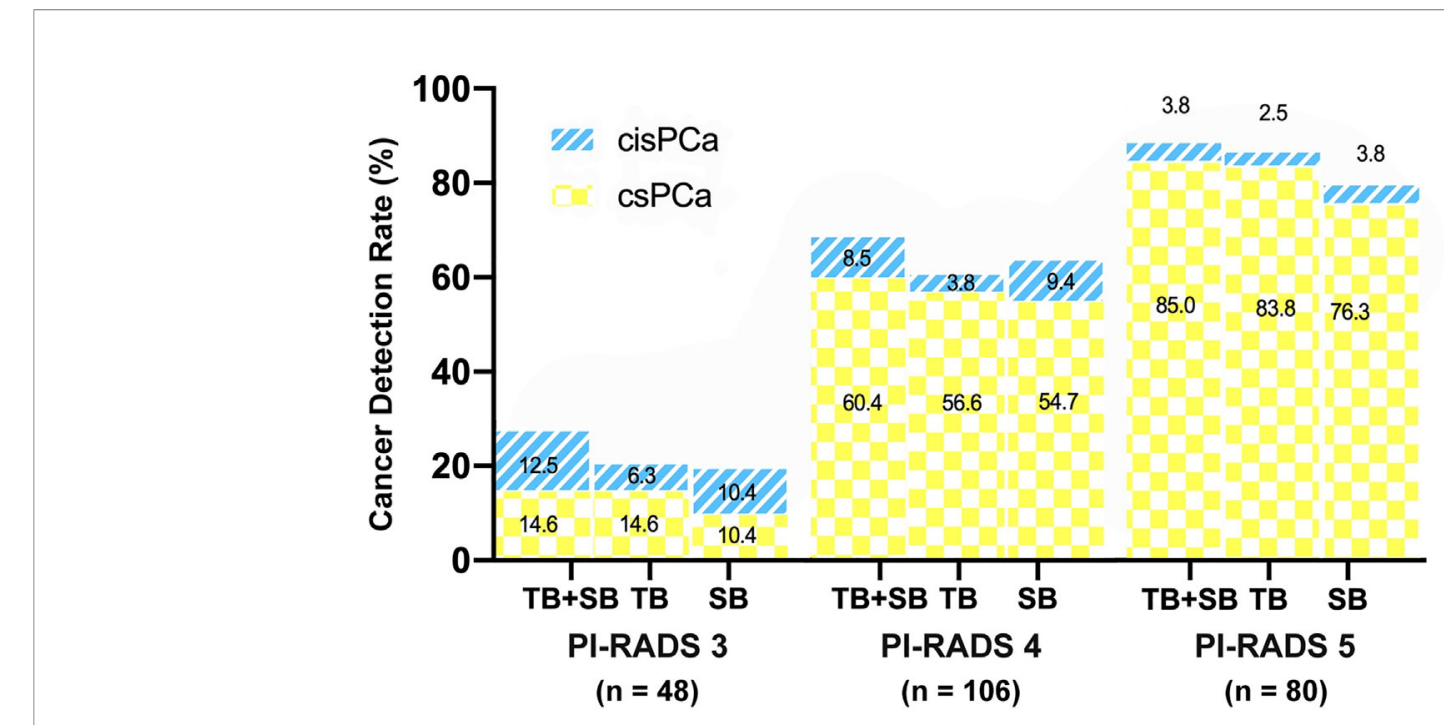

FIGURE 3 | Comparison of csPCa (GS $\geq 7$ ) and cisPCa (GS6) cancer detection between TB+SB, TB alone, and SB alone stratified by PI-RADS score. 


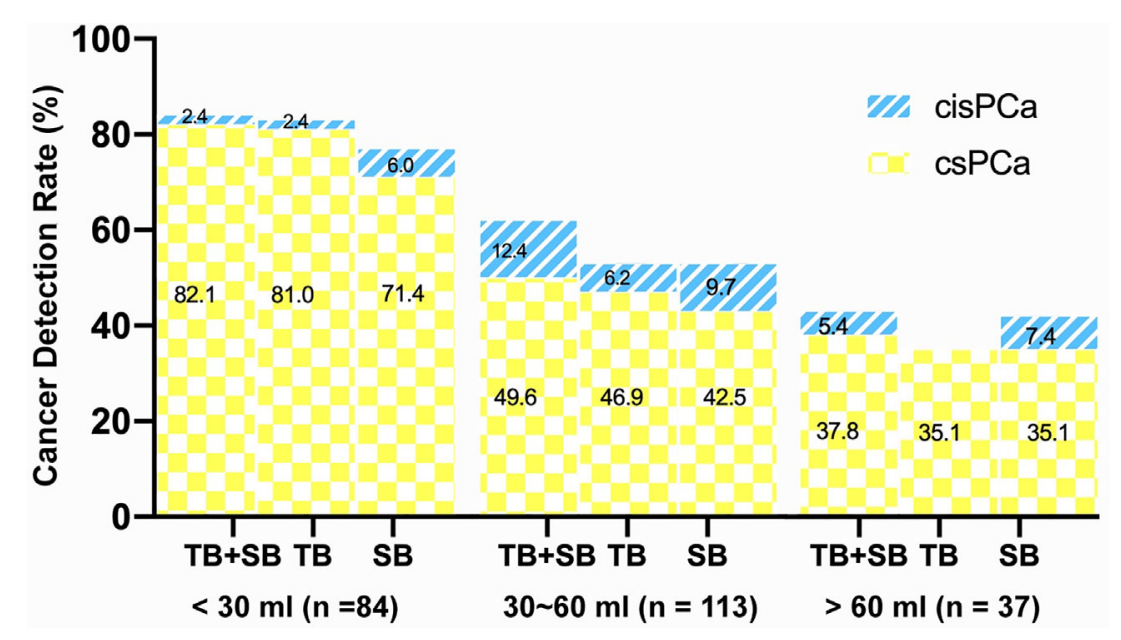

FIGURE 4 | Comparison of csPCa (GS $\geq 7$ ) and cisPCa (GS6) cancer detection between TB+SB, TB alone, and SB alone stratified by prostate volume.

TABLE 3 | Characteristics of the study population stratified by PI-RADS.

\begin{tabular}{lccc}
\hline Parameter & PI-RADS 5 & PI-RADS 3-4 & P \\
\hline Number & 80 & 154 & \\
Age & $68.5 \pm 9.35$ & $65.1 \pm 8.54$ & 0.232 \\
PSA & $16.8 \pm 13.84$ & $10.3 \pm 5.89$ & 0.000 \\
Prostate volume & $39.7 \pm 18.5$ & $43.5 \pm 25.89$ & 0.073 \\
csPCa detection (\%) & & & \\
TB+SB & $68(85 \%)$ & $71(46.1 \%)$ & 0.000 \\
TB alone & $67(83.8 \%)$ & $67(43.5 \%)$ & 0.000 \\
SB alone & $61(76.3 \%)$ & $63(40.9 \%)$ & 0.000 \\
GG concordant between TB and SB & $47(66.2 \%)$ & $58(67.4 \%)$ & 0.869 \\
GG discordant between TB and SB & $24(33.8 \%)$ & $28(32.6 \%)$ & - \\
$\quad$ Upgrading on TB & $19(79.2 \%)$ & $14(50 \%)$ & 0.029 \\
$\quad$ Upgrading on SB & $5(20.8 \%)$ & $14(50 \%)$ & 0.029 \\
Radical prostatectomy & 50 & 55 & \\
$\quad$ Upgrading over TB+SB & $3(6 \%)$ & $8(14.5 \%)$ & 0.153 \\
Upgrading over TB & $3(6 \%)$ & $14(25.5 \%)$ & 0.003 \\
Upgrading over SB & $14(28 \%)$ & $12(21.8 \%)$ & 0.464 \\
\hline
\end{tabular}

PI-RADS 5 group $(83.8$ vs $76.3 \%, \mathrm{P}=0.07)$ or in PI-RADS 3-4 group (43.5 vs $40.9 \%, \mathrm{P}=1.0)$.

With respect to the GG concordance between $\mathrm{TB}$ and $\mathrm{SB}$, the concordant rate was similar in PI-RADS 5 and PI-RADS 3-4 subgroup (66.2 vs 67.4\%, P =0.869, Table 3). Patients with PIRADS 5 lesions were more likely to experience upgrading on TB than PI-RADS 3-4 patients (79.2 vs 50\%, $\mathrm{P}=0.029)$. Conversely, patients with PI-RADS 3-4 lesions were more likely to experience upgrading on SB compared to PI-RADS 5 patients (50 vs 20.8\%, $\mathrm{P}=0.029$ ).

\section{Targeted Versus Systematic Cancer Detection and Risk Stratification Stratified by Prostate Volume}

When the whole cohort was stratified by prostate volume (PV), $\mathrm{TB}$ alone detected significantly more csPCa than SB in small prostate $(\mathrm{PV}<30 \mathrm{ml})$ group $(81.0$ vs $71.0 \%, \mathrm{P}=0.021)$ but not in large prostate $(\mathrm{PV} \geq 30 \mathrm{ml}$ ) group ( $44 v s 42.7 \%, \mathrm{P}=0.754$ ). The conjunction with SB detected additional one and four csPCa in small and large prostate group respectively, but didn't increase the csPCa detection rate compared with $\mathrm{TB}$ alone in each subgroup (82.1 vs $81 \%, \mathrm{P}=1.0 ; 46.7$ vs $44 \%, \mathrm{P}=0.125$ ).

As for GG concordance between the two methods, men with small prostate seems more likely to experience upgrading on TB than men with large prostate (78.9 vs $54.5 \%$, Table 4 ), but the difference was of no significance $(\mathrm{P}=0.078)$.

\section{Pathological Concordance on Radical Prostatectomy}

Out of 157 prostate cancer patients detected by combined biopsy, 105 subsequently underwent radical prostatectomy (RP) at our institution. In total, 11 (10.5\%) of 105 patients had upgraded GG on RP. There were four men upgraded from GG1 to GG2 $(n=4)$, two from GG2 to GG3, one from GG3 to GG4, and two from GG3 to GG5. The other two patients were misdiagnosed as no cancer in

TABLE 4 | Characteristics of the study population stratified by prostate volume.

\begin{tabular}{lccc}
\hline Parameter & PV $<\mathbf{3 0} \mathbf{~} \mathbf{l}$ & $\mathbf{P V} \geq \mathbf{3 0} \mathbf{~ m l}$ & $\mathbf{P}$ \\
\hline Number & 84 & 150 & \\
Age & $63.3 \pm 8.43$ & $68.0 \pm 8.80$ & 0.354 \\
PSA & $12.1 \pm 9.57$ & $12.8 \pm 10.16$ & 0.649 \\
PI-RADS & & & \\
3 & $8(9.5 \%)$ & $40(26.7 \%)$ & 0.008 \\
4 & $43(51.2 \%)$ & $63(42 \%)$ & \\
5 & $33(39.3 \%)$ & $47(31.3 \%)$ & \\
CSPCa detection (\%) & & & \\
TB+SB & $69(82.1 \%)$ & $70(46.7 \%)$ & 0.000 \\
TB alone & $68(81 \%)$ & $66(44 \%)$ & 0.000 \\
SB alone & $60(71 \%)$ & $64(42.7 \%)$ & 0.000 \\
GG concordant between TB and SB & $52(73.2 \%)$ & $53(61.6 \%)$ & 0.124 \\
GG disconcordant between TB and SB & $19(26.8 \%)$ & $33(38.4 \%)$ & 0.124 \\
Upgrading on TB & $15(78.9 \%)$ & $18(54.5 \%)$ & 0.078 \\
$\quad$ Upgrading on SB & $4(21.1 \%)$ & $15(45.5 \%)$ & 0.078 \\
Radical prostatectomy & 47 & 58 & \\
Upgrading over TB+SB & $3(6.4 \%)$ & $8(13.8 \%)$ & 0.218 \\
Upgrading over TB & $4(8.5 \%)$ & $13(22.4 \%)$ & 0.054 \\
Upgrading over SB & $7(14.9 \%)$ & $19(32.8 \%)$ & 0.035 \\
$\quad$ & &
\end{tabular}


initial biopsy but detected GG2 cancer in the second biopsy and subsequent radical prostatectomy, therefore upgraded from no cancer to GG2. Either TB alone or SB alone would lead 17 (16.2\%) and 26 (24.8\%) men upgraded on RP respectively, with a significantly greater rate of disease upgrading than combined biopsy ( $\mathrm{P}=0.031, \mathrm{P}<0.001$ respectively). Difference in rates of upgrading between $\mathrm{TB}$ alone and $\mathrm{SB}$ alone was not significant $(\mathrm{P}=0.078)$.

When stratified by PI-RADS score, patients in PI-RADS 3-4 group had significant greater GG upgrading on RP over TB than PI-RADS 5 group (25.5 vs 6\%, $\mathrm{P}=0.003)$. The omission of SB would lead six more patients' Gleason score upgraded on RP in PI-RADS 3-4 group, but not lead any disease upgrading in PIRADS 5 group as compared to combined biopsy (Table 3). In other words, combined biopsy was superior to TB alone in PIRADS 3-4 patients (14.5 vs 25.5\%, $\mathrm{P}=0.031$ ), but was no superior to TB alone in PI-RADS 5 patients ( 6 vs $6 \%, \mathrm{P}=1.0$ ) for decreasing the rate of Gleason upgrading on RP. TB had a significantly lower rate disease upgrading on RP than SB in PIRADS 5 group ( 6 ss $28 \%, \mathrm{P}=0.001$ ), and a similar upgrading rate with SB in PI-RADS 3-4 group (25.5 vs 21.8\%, $\mathrm{P}=0.754$ ).

As stratified by prostate volume, men with large prostate seem more likely to experience disease upgrading on $\mathrm{RP}$ than small prostate by any of $\mathrm{TB}+\mathrm{SB}, \mathrm{TB}$, or $\mathrm{SB}$, but with no significant difference except for SB (Table 4). The addition of SB would not significantly decrease the upgrading rate on RP compared with TB alone in either small prostate group (6.4 vs $8.5 \%, \mathrm{P}=1.0)$ or large prostate group ( 13.8 vs $22.4 \%, \mathrm{P}=0.063$ ). Also, the upgrading rate of TB was not significantly lower than that of SB in each group (8.5 vs $14.9 \%, \mathrm{P}=0.21 ; 22.4$ vs $32.8 \%, \mathrm{P}=0.375$ for small and large prostate group respectively). However, combined biopsy significantly decreased the risk of disease upgrading on RP compared with SB alone in large prostate group (13.8 vs 32.8\%, $\mathrm{P}=0.001)$ other than small prostate group (6.4 vs $14.9 \%, \mathrm{P}=0.125)$. These data suggest that those who have large prostate would benefit from combined biopsy in reducing disease upgrading on RP.

\section{DISCUSSION}

There has been considerable concern regarding whether additional systematic biopsy is required for MRI-visible lesions (13, 20-22). Several previous studies demonstrated that the combination of MRI-targeted and systematic biopsy obtain the maximal detection of csPCa than either biopsy method alone $(21,23)$. Systematic biopsy beneficially detected additional $3.5-13 \%$ csPCa in MRIpositive men (24), while approximately $6 \%$ csPCa had the risk stratification upgrading by systematic biopsy over targeted biopsy (25). In the present study, cognitive MRI-guided targeted biopsy showed greater csPCa detection rate and lower cisPCa detection rate with greater percentage of cancer involvement than systematic biopsy. Although systematic biopsy beneficially detected additional $6 \%$ prostate cancers in the whole cohort, only $28.6 \%$ of them were csPCa. The additional systematic biopsy indicated only a marginal increase of csPCa detection but a remarkable increase of cisPCa detection compared with targeted biopsy in the whole study population. While, the rate of Gleason upgrading on RP was lowest in combined biopsy (6.4\%), followed by TB (8.5\%) and SB (14.9\%). This raises the question about the necessity of performing systematic biopsy among all patients with MRI-visible lesions.

It has been reported that the utility of MRI/US fusiontargeted biopsy was especially relevant at enlarged prostate, higher MRI suspicious and higher PSA level (21, 26-28). However, few of the previous studies indicate if systematic biopsy can be omitted dependent on these variables. To our knowledge, this is the first study to evaluate the performance of additional systematic biopsy in biopsy naïve patients taking PIRADS scores and prostate volume into consideration.

PI-RADS score was suggested as a predict factor of csPCa. In this study, we found that the probability of csPCa detection increased as PI-RADS score increased (Figure 3), which was consistent with earlier studies $(21,26)$. When stratified by PIRADS score, GG upgrading on targeted biopsy was more commonly observed in PI-RADS 5 subgroup, while GG upgrading on systematic biopsy was more commonly observed in PI-RADS 3-4 subgroup. Among patients with PI-RADS 3-4 lesions, they had equal probability of upgrading on either targeted or systematic biopsy. Moreover, the combined biopsy method showed the same rate of disease upgrading on radical prostatectomy in PI-RADS 5 subgroup, and a significantly decreased upgrading rate in PI-RADS 3-4 subgroup, as compared to targeted biopsy alone. These findings suggest that additional systematic biopsy could be avoided in patients with PI-RADS 5 lesions, but is required for accurate risk stratification of prostate cancer in patients with PI-RADS 3-4 lesions. Previously, Ahmad et al.'s study also came to the similar findings in a repeated biopsy cohort study (29).

Prostate volume is another important variable taken into consideration for prostate biopsy decision $(30,31)$. The risk of sampling error for systematic biopsy increased, thus resulted in lower detection rate of prostate cancer as prostate volume increased. Large prostate volume has also been reported to be associated with disease upgrading based by systematic biopsy over MRI/US Fusion-guided targeted biopsy due to increased operator-dependent deformation during the biopsy procedure (25). Herein, we found that both targeted and systematic biopsy had a decreased csPCa detection rate as prostate volume increased (Figure 4), which is consistent with prior findings $(21,27)$. Among those cases with discordant GG between the two biopsies, men with large volume prostate seem more likely to be upgraded by systematic biopsy over targeted biopsy. Prior study by De et al. showed that MRI/US fusion-guided targeted biopsy outperformed systematic biopsy for detection of csPCa in large volume prostate $(>40 \mathrm{ml}$ ) compared to smaller volume prostate and suggested systematically perform MRI-US fusion biopsies rather than systematic biopsies as a first line approach in prostate volume greater than $40 \mathrm{ml}$ (27). But the present study showed that cognitive MRI-guided targeted biopsy showed significantly higher csPCa detection rate (81\%) than systematic biopsy (71\%) in small volume prostate $(<30 \mathrm{ml})$, rather than in large volume prostate $(\geq 30 \mathrm{ml})$. This result was in consistent with Wysock et al.'s study which demonstrated that smaller prostate volume was a predicted factor of increased cancer detection rate on 
targeted biopsy, likely because of decreased sampling error and needle deflection (32). Moreover, we also found that disease upgrading on radical prostatectomy over each biopsy was more commonly observed in large prostate than small prostate. Notably, the distribution of PI-RADS score was not equal between small and large PV group due to the prevalence of csPCa, which may make a potential effect on these results. These findings suggest that the additional systematic biopsy benefit more in large prostate than small prostate, which could not be omitted in men with large prostate $(\geq 30 \mathrm{ml})$.

This study has several limitations. Firstly, this is retrospective study in a large referral institution, which may lead potential selection biases. Secondly, lesion-to-lesion comparison in patients with multiple MRI suspicious lesions was not available in the study. The comparisons of biopsy methods were performed per patient rather than per lesion. Thirdly, we used cognitive MRI-guided targeted biopsy instead of MRI/US fusion targeted biopsy, which may reduce the performance of MRI guided targeted biopsy. But all the cognitive targeted prostate biopsies were performed by two experienced urologists and guided by an experienced urological radiologist. The findings should be further validated in large, prospective, multi-center studies.

In conclusion, the combination biopsy method was no superior than targeted biopsy alone in PI-RADS 5 or in small volume prostate subgroup in initial prostate biopsy patients with abnormal MRI. Large volume prostate $(\geq 30 \mathrm{ml})$ and lower PIRADS categories (PI-RADS 3-4) may benefit more from the addition of systematic biopsy than the converse.

\section{REFERENCES}

1. Smeenge $\mathrm{M}$, de la Rosette JJ, Wijkstra H. Current status of transrectal ultrasound techniques in prostate cancer. Curr Opin Urol (2012) 22(4):297302. doi: 10.1097/MOU.0b013e3283548154

2. Bjurlin MA, Carter HB, Schellhammer P, Cookson MS, Gomella LG, Troyer $\mathrm{D}$, et al. Optimization of initial prostate biopsy in clinical practice: sampling, labeling and specimen processing. J Urol (2013) 189(6):2039-46. doi: 10.1016/ j.juro.2013.02.072

3. Siddiqui MM, Rais-Bahrami S, Truong H, Stamatakis L, Vourganti S, Nix J, et al. Magnetic Resonance Imaging/Ultrasound-Fusion Biopsy Significantly Upgrades Prostate Cancer Versus Systematic 12-core Transrectal Ultrasound Biopsy. Eur Urol (2013) 64(5):713-9. doi: 10.1016/j.eururo.2013.05.059

4. Ahmed HU, El-Shater Bosaily A, Brown LC, Gabe R, Kaplan R, Parmar MK, et al. Diagnostic accuracy of multi-parametric MRI and TRUS biopsy in prostate cancer (PROMIS): a paired validating confirmatory study. Lancet (2017) 389(10071):815-22. doi: 10.1016/S0140-6736(16)32401-1

5. Bjurlin MA, Taneja SS. Prediagnostic Risk Assessment with Prostate MRI and MRI-Targeted Biopsy. Urol Clin North Am (2017) 44(4):535-46. doi: 10.1016/ j.ucl.2017.07.012

6. Patel U, Dasgupta P, Challacombe B, Cahill D, Brown C, Patel R, et al. Prebiopsy 3-Tesla MRI and targeted biopsy of the index prostate cancer: correlation with robot-assisted radical prostatectomy. BJU Int (2017) 119 (1):82-90. doi: 10.1111/bju.13525

7. Tonttila PP, Lantto J, Pääkkö E, Piippo U, Kauppila S, Lammentausta E, et al. Prebiopsy Multiparametric Magnetic Resonance Imaging for Prostate Cancer Diagnosis in Biopsy-naive Men with Suspected Prostate Cancer Based on Elevated Prostate-specific Antigen Values: Results from a Randomized Prospective Blinded Controlled Trial. Eur Urol (2016) 69(3):419-25. doi: 10.1016/j.eururo.2015.05.024

\section{DATA AVAILABILITY STATEMENT}

The raw data supporting the conclusions of this article will be made available by the authors, without undue reservation.

\section{ETHICS STATEMENT}

The studies involving human participants were reviewed and approved by the Institutional Review Board of West China Hospital of Sichuan University. Written informed consent for participation was not required for this study in accordance with the national legislation and the institutional requirements.

\section{AUTHOR CONTRIBUTIONS}

$\mathrm{XC}$ and YC designed the study. GS and ZL acquired the data. LY and JY analyzed and interpreted the data. XC and JX wrote the manuscript. HZ and BS reviewed the manuscript. All authors contributed to the article and approved the submitted version.

\section{FUNDING}

This work was funded by Science and Technology Innovation Talent of Sichuan (20CXRC0065) and Science and Technology Project of Chengdu(2019-YF05-00376-SN).

8. Radtke JP, Schwab C, Wolf MB, Freitag MT, Alt CD, Kesch C, et al. Multiparametric Magnetic Resonance Imaging (MRI) and MRI-Transrectal Ultrasound Fusion Biopsy for Index Tumor Detection: Correlation with Radical Prostatectomy Specimen. Eur Urol (2016) 70(5):846-53. doi: 10.1016/j.eururo.2015.12.052

9. Kasivisvanathan V, Rannikko AS, Borghi M, Panebianco V, Mynderse LA, Vaarala MH, et al. MRI-Targeted or Standard Biopsy for Prostate-Cancer Diagnosis. New Engl J Med (2018) 378(19):1767-77. doi: 10.1056/ NEJMoa1801993

10. Rouvière $\mathrm{O}$, Puech $\mathrm{P}$, Renard-Penna R, Claudon M, Roy C, Mège-Lechevallier $\mathrm{F}$, et al. Use of prostate systematic and targeted biopsy on the basis of multiparametric MRI in biopsy-naive patients (MRI-FIRST): a prospective, multicentre, paired diagnostic study. Lancet Oncol (2019) 20(1):100-9. doi: 10.1016/S1470-2045(18)30569-2

11. Woo S, Suh CH, Eastham JA, Zelefsky MJ, Morris MJ, Abida W, et al. Comparison of Magnetic Resonance Imaging-stratified Clinical Pathways and Systematic Transrectal Ultrasound-guided Biopsy Pathway for the Detection of Clinically Significant Prostate Cancer: A Systematic Review and Metaanalysis of Randomized Controlled Trials. Eur Urol Oncol (2019) 2(6):605-16. doi: 10.1016/j.euo.2019.05.004

12. Delongchamps NB, Peyromaure M, Schull A, Beuvon F, Bouazza N, Flam T, et al. Prebiopsy Magnetic Resonance Imaging and Prostate Cancer Detection: Comparison of Random and Targeted Biopsies. J Urol (2013) 189(2):493-9. doi: 10.1016/j.juro.2012.08.195

13. van der Leest M, Cornel E, Israël B, Hendriks R, Padhani AR, Hoogenboom M, et al. Head-to-head Comparison of Transrectal Ultrasound-guided Prostate Biopsy Versus Multiparametric Prostate Resonance Imaging with Subsequent Magnetic Resonance-guided Biopsy in Biopsy-naïve Men with Elevated Prostate-specific Antigen: A Large Prospective Multicenter Clinical Study. Eur Urol (2019) 75(4):570-8. doi: 10.1016/j.eururo.2018.11.023 
14. Schoots IG, Roobol MJ, Nieboer D, Bangma CH, Steyerberg EW, Hunink MG. Magnetic Resonance Imaging-targeted Biopsy May Enhance the Diagnostic Accuracy of Significant Prostate Cancer Detection Compared to Standard Transrectal Ultrasound-guided Biopsy: A Systematic Review and Metaanalysis. Eur Urol (2015) 68(3):438-50. doi: 10.1016/j.eururo.2014.11.037

15. Mottet N, van den Bergh RCN, Briers E, Van den Broeck T, Cumberbatch MG, De Santis M, et al. EAU-EANM-ESTRO-ESUR-SIOG Guidelines on Prostate Cancer-2020 Update. Part 1: Screening, Diagnosis, and Local Treatment with Curative Intent. Eur Urol (2021) 79(2):243-62. doi: 10.1016/j.eururo.2020.09.042

16. NICE Guidance. Prostate cancer: diagnosis and management: (c) NICE (2019) Prostate cancer: diagnosis and management. BJU Int (2019) 124(1):9-26. doi: 10.1111/bju.14809

17. Siddiqui MM, Rais-Bahrami S, Turkbey B, George AK, Rothwax J, Shakir N, et al. Comparison of MR/Ultrasound Fusion-Guided Biopsy With Ultrasound-Guided Biopsy for the Diagnosis of Prostate Cancer. Jama (2015) 313(4):390-7. doi: 10.1001/jama.2014.17942

18. Weinreb JC, Barentsz JO, Choyke PL, Cornud F, Verma S. PI-RADS Prostate Imaging - Reporting and Data System: 2015, Version 2. Eur Urol (2015) 69 (1):16-40. doi: 10.1016/j.eururo.2015.08.052

19. Epstein JI, Egevad L, Amin MB, Delahunt B, Srigley JR, Humphrey PA, et al. The 2014 International Society of Urological Pathology (ISUP) Consensus Conference on Gleason Grading of Prostatic Carcinoma: Definition of Grading Patterns and Proposal for a New Grading System. Am J Surg Pathol (2016) 29:244-52. doi: 10.1097/PAS.0000000000000530

20. Barentsz J, Futterer JJ, Padhani AR. Will Magnetic Resonance Imaging-guided Biopsy Replace Systematic Biopsy? Eur Urol Focus (2015) 1(2):152-5. doi: 10.1016/j.euf.2015.07.008

21. Elkhoury FF, Felker ER, Kwan L, Sisk AE, Delfin M, Natarajan S, et al. Comparison of Targeted vs Systematic Prostate Biopsy in Men Who Are Biopsy Naive: The Prospective Assessment of Image Registration in the Diagnosis of Prostate Cancer (PAIREDCAP) Study. JAMA Surg (2019) 154 (9):811-8. doi: 10.1001/jamasurg.2019.1734

22. Marra G, Ploussard G, Futterer J, Valerio M, Party E.-Y.P.C.W. Controversies in MR targeted biopsy: alone or combined, cognitive versus software-based fusion, transrectal versus transperineal approach? World J Urol (2019) 37 (2):277-87. doi: 10.1007/s00345-018-02622-5

23. Lee AYM, Yang XY, Lee HJ, Law YM, Huang HH, Lau WKO, et al. Multiparametric MRI-ultrasonography software fusion prostate biopsy: initial results using a stereotactic robotic-assisted transperineal prostate biopsy platform comparing systematic vs targeted biopsy. BJU Int (2020) 126(5):568-76. doi: 10.1016/S2666-1683(20)34155-0

24. Schoots IG, Padhani AR, Rouvière O, Barentsz JO, Richenberg J. Analysis of Magnetic Resonance Imaging-directed Biopsy Strategies for Changing the Paradigm of Prostate Cancer Diagnosis. Eur Urol Oncol (2019) 3(1):32-41. doi: 10.1016/j.euo.2019.10.001
25. Muthigi A, George AK, Sidana A, Kongnyuy M, Simon R, Moreno V, et al. Missing the Mark: Prostate Cancer Upgrading by Systematic Biopsy over Magnetic Resonance Imaging/Transrectal Ultrasound Fusion Biopsy. J Urol (2017) 197(2):327-34. doi: 10.1016/j.juro.2016.08.097

26. Walton Diaz A, Hoang AN, Turkbey B, Hong CW, Truong H, Sterling T, et al. Can magnetic resonance-ultrasound fusion biopsy improve cancer detection in enlarged prostates? J Urol (2013) 190(6):2020-5. doi: 10.1016/j.juro.2013.05.118

27. de Gorski A, Rouprêt M, Peyronnet B, Le Cossec C, Granger B, Comperat E, et al Accuracy of Magnetic Resonance Imaging/Ultrasound Fusion Targeted Biopsies to Diagnose Clinically Significant Prostate Cancer in Enlarged Compared to Smaller Prostates. J Urol (2015) 194(3):669-73. doi: 10.1016/j.juro.2015.03.025

28. Shakir NA, George AK, Siddiqui MM, Rothwax JT, Rais-Bahrami S, Stamatakis L, et al. Identification of Threshold Prostate Specific Antigen Levels to Optimize the Detection of Clinically Significant Prostate Cancer by Magnetic Resonance Imaging/Ultrasound Fusion Guided Biopsy. J Urol (2014) 192(6):1642-9. doi: 10.1016/j.juro.2014.08.002

29. Arabi A, Deebajah M, Yaguchi G, Pantelic M, Williamson S, Gupta N, et al. Systematic Biopsy Does Not Contribute to Disease Upgrading in Patients Undergoing Targeted Biopsy for PI-RADS 5 Lesions Identified on Magnetic Resonance Imaging in the Course of Active Surveillance for Prostate Cancer. Urology (2019) 134:168-72. doi: 10.1016/j.urology.2019.08.035

30. Xu N, Wu YP, Li XD, Lin MY, Zheng QS, Chen SH, et al. Risk of upgrading from prostate biopsy to radical prostatectomy pathology: Is magnetic resonance imaging-guided biopsy more accurate? J Cancer (2018) 9 (19):3634-9. doi: 10.7150/jca.26791

31. Freedland SJ, Isaacs WB, Platz EA, Terris MK, Aronson WJ, Amling CL, et al. Prostate size and risk of high-grade, advanced prostate cancer and biochemical progression after radical prostatectomy: a search database study. J Clin Oncol (2005) 23(30):7546-54. doi: 10.1200/JCO.2005.05.525

32. Wysock JS, Rosenkrantz AB, Huang WC, Stifelman MD, Lepor H, Deng FM, et al. A prospective, blinded comparison of magnetic resonance (MR) imaging-ultrasound fusion and visual estimation in the performance of MR-targeted prostate biopsy: the PROFUS trial. Eur Urol (2014) 66(2):34351. doi: $10.1016 /$ j.eururo.2013.10.048

Conflict of Interest: The authors declare that the research was conducted in the absence of any commercial or financial relationships that could be construed as a potential conflict of interest.

Copyright (C) 2021 Cheng, Xu, Chen, Liu, Sun, Yang, Yao, Zeng and Song. This is an open-access article distributed under the terms of the Creative Commons Attribution License (CC BY). The use, distribution or reproduction in other forums is permitted, provided the original author(s) and the copyright owner(s) are credited and that the original publication in this journal is cited, in accordance with accepted academic practice. No use, distribution or reproduction is permitted which does not comply with these terms. 